\title{
Uncertainty-Based Approach for Dynamic Aerodynamic Data Acquisition and Analysis
}

\author{
Eugene H. D. Heim ${ }^{*}$ and Jay M. Bandon ${ }^{\dagger}$ \\ NASA Langley Research Center, Hampton, Virginia, 23681
}

\begin{abstract}
Development of improved modeling methods to provide increased fidelity of flight predictions for aircraft motions during flight in flow regimes with large nonlinearities requires improvements in test techniques for measuring and characterizing wind tunnel data. This paper presents a method for providing a measure of data integrity for static and forced oscillation test techniques. Data integrity is particularly important when attempting to accurately model and predict flight of today's high performance aircraft which are operating in expanded flight envelopes, often maneuvering at high angular rates at high angles-of-attack, even above maximum lift. Current aerodynamic models are inadequate in predicting flight characteristics in the expanded envelope, such as rapid aircraft departures and other unusual motions. Present wind tunnel test methods do not factor changes of flow physics into data acquisition schemes, so in many cases data are obtained over more iterations than required, or insufficient data may be obtained to determine a valid estimate with statistical significance. Additionally, forced oscillation test techniques, one of the primary tools used to develop dynamic models, do not currently provide estimates of the uncertainty of the results during an oscillation cycle. A method to optimize the required number of forced oscillation cycles based on decay of uncertainty gradients and balance tolerances is also presented.
\end{abstract}

\section{Nomenclature}

Amp. $\quad=$ amplitude of oscillation in degrees

$b=$ wing span in feet

$B_{i} \quad=$ estimate of systematic error for the $i^{\text {th }}$ variable in the generic function $r$ given with $95 \%$ confidence

$B_{i k} \quad=$ estimate of the covariance of the systematic error

$B_{r} \quad=$ estimate of systematic error in the generic function $r$ given with $95 \%$ confidence

$\bar{c} \quad=$ mean aerodynamic chord in feet

$C_{l} \quad=$ rolling moment coefficient

$C_{m} \quad=$ pitching moment coefficient

$C_{N} \quad=$ normal force coefficient

$C_{n} \quad=$ yawing moment coefficient

$f \quad=$ frequency of oscillation in $\mathrm{Hz}$

$g_{m} \quad=10$-cycle moving average of the maximum gradient of uncertainty from cycle to cycle of the $n$ interpolated points around the oscillation cycle

$i, j, k=$ generic indices

$J \quad=$ total number of variables in generic function $r$

$n=$ number of interpolated points around a forced oscillation cycle

$N \quad=$ number of solutions to the generic function $r$

$q \quad=$ dynamic pressure in pounds per square foot

$Q \quad=$ number of variables in the generic function $r$ that have correlated systematic error sources

$r \quad=$ generic function

$r_{k}=k^{\text {th }}$ solution of the generic function $r$

$S=$ reference area in square feet

\footnotetext{
* Aerospace Engineer, Vehicle Dynamics Branch, 18C West Taylor Street/MS 153

$\dagger$ Aerospace Engineer, Vehicle Dynamics Branch, 18C West Taylor Street/MS 153, Associate Fellow
}

1

American Institute of Aeronautics and Astronautics 


$\begin{array}{lll}\bar{r} & =\text { mean value of multiple solutions of the generic function } r \\ S_{r} & = & \text { standard deviation of multiple solutions of the generic function } r \\ S_{\bar{r}} & = & \text { standard deviation of the mean of solutions of the generic function } r \\ U_{r} & = & \text { total uncertainty estimate of the generic function } r \text { given with } 95 \% \text { confidence } \\ U_{\bar{r}} & = & \text { total uncertainty estimate of the mean of multiple solutions to the generic function } r \text { given with } 95 \% \\ & \quad \text { confidence } \\ x & =\text { reading of subject under measurement } \\ x_{\text {true }} & =\text { actual value of subject under measurement } \\ \alpha & =\text { angle of attack given in degrees } \\ \beta & =\text { bias error, systematic component of the total error } \\ \delta & =\text { total error, difference between a reading and the true value of a measurement } \\ \varepsilon & =\text { precision error, random and unsteady component of the total error } \\ \theta & =\text { angular position in oscillation cycle } \\ \mu & \text { mean value of a Gaussian distribution }\end{array}$

\section{Introduction}

$\mathrm{T}$

ODAY'S high performance aircraft are operating in expanded flight envelopes, often maneuvering at high angular rates at high angles-of-attack, even above maximum lift. Current aerodynamic models are often inadequate in predicting flight characteristics in the expanded envelope, such as rapid aircraft departures and other unusual motions. Modern control theory includes methods that can adequately define a robust control system in the presence of known uncertainty boundaries. ${ }^{1}$ This requires accurate models obtained from either testing or calculations to adequately predict flight dynamic behavior. As modeling methods improve to provide increased fidelity flight predictions during flight in flow regimes with large nonlinearities, improvements in test techniques for measuring and characterizing the data will be critical. Testing in the presence of unsteady flow conditions presents a challenge not only for modeling, but also for determining bounds on data knowledge/reliability or data integrity.

Dynamic data, in particular, has not been well quantified in past studies as to the reliability of the results. The uncertainty boundaries are certainly a function of unsteady flow characteristics, systematic instrumentation errors, measurement precision, and also mathematical model formulation. The math model used to describe the data is an area of research currently underway, and use of the raw data to develop new modeling approaches needs some quantification of how reliable the measurements are. Present wind tunnel test methods do not factor changes of flow physics into data acquisition schemes, so in many cases data are obtained over more iterations than required, or insufficient data may be obtained to determine a valid estimate of the forces and moments on a model with any statistical significance. Additionally, forced oscillation test techniques, one of the primary tools used to develop dynamic models, do not currently provide estimates of the uncertainty of the results during an oscillation cycle. Currently data from multiple cycles are recorded and then ensemble averaged to provide a smoothed estimate of the dynamic aerodynamic characteristics. The number of cycles needed to provide a valid representation of aerodynamic characteristics has typically been arrived at through trial and error. A fixed number of cycles has then been used for the entire envelop of testing. This ad-hoc process almost certainly results in longer test times for some conditions and shorter than required for others.

This paper presents a method for providing a measure of data integrity for static and forced oscillation test techniques. The theory of propagation of uncertainties is employed to arrive at a quantitative and qualitative measure of data integrity and is briefly reviewed in the subsequent section followed by a discussion of general error sources and paths considered. ${ }^{2,3}$ Examples and a brief discussion of data integrity for static and dynamic wind tunnel data are then be presented. Finally, a method for optimizing the required number of cycles of a forced oscillation test based on decay of uncertainty gradients of body axis coefficients and a user supplied threshold is proposed.

\section{Propagation of Uncertainties}

Error is an inherent part of every measurement. The use of experimental data in an analytical solution requires an understanding of the experimental uncertainties involved. The difference between a measurement and the true value, $x_{\text {true }}$ is defined as the total error, $\delta$. The total error consists of a systematic component, referred to as the bias error, $\beta$ and a random component or precision error, $\varepsilon$. In cases where the variations in the readings come from many small random errors each of equal magnitude, with each error being just as likely to be negative as positive, the smooth distribution of an infinite number of readings typically coincides with the Gaussian distribution. The Gaussian distribution has been shown to describe more experimental and instrumentation variation than any other statistical 
distribution. The difference between the mean of the Gaussian distribution, $\mu$ and the true value is equal to the bias, so for any single reading, $x$ the total error is

$$
\delta=x-x_{\text {true }}=\beta+\varepsilon
$$

Coleman and Steele have used this relationship along with a first order Taylor series expansion of the variance of a generic function, $r$ composed of $J$ variables such that

$$
r=r\left(x_{1}, x_{2}, \ldots, x_{J}\right)
$$

to estimate how systematic and random errors from individual measured variables propagate through the function to yield an uncertainty of the final result. ${ }^{2}$ This method of propagating known errors is used in this paper primarily to propagate bias errors from instrumentation, calibrations and tare interpolations (discussed later) through the body axis coefficient data reduction equations. Correlated systematic errors, biases in different variables that arise from the same source, are of major concern as they can have significant impacts on the uncertainty estimations. The systematic error of the result, $B_{r}$ is given from Ref. 2 as

$$
B_{r}=\sqrt{\sum_{i=1}^{J}\left(\frac{\partial r}{\partial x_{i}}\right)^{2} B_{i}^{2}+2 \sum_{i=1}^{J-1} \sum_{k=i+1}^{J}\left(\frac{\partial r}{\partial x_{i}}\right)\left(\frac{\partial r}{\partial x_{k}}\right) B_{i k}}
$$

where $B_{i}$ is an estimate of the systematic error from each variable with an inherent assumed $95 \%$ confidence and the estimate of the covariance of the systematic error, $B_{i k}$ is approximated by

$$
B_{i k}=\sum_{j=1}^{Q}\left(B_{i}\right)_{j}\left(B_{k}\right)_{j}
$$

for $Q$ correlated sources. Analytical determinations of the partial derivatives of higher order polynomial regressions are prohibitive especially with multiple independent variables and numerous data pairs. Numerical approximations are typically used in such situations.

Random errors are traditionally defined as errors that do not correlate with anything, such as broad-band white noise. Structural dynamics, vortex shedding, the wind tunnel fan beat frequency, etc. along with white noise are several sources of variation in wind tunnel data. Many of these sources could be correlated with the signal content from the balance and other instrumentation. The term "random" will be used loosely to define all content that is unsteady or not fixed.

The random error contribution to the uncertainty is determined directly from the result. For example consider a result that is determined $N$ times. The average would be

$$
\bar{r}=\frac{1}{N} \sum_{k=1}^{N} r_{k}
$$

and a standard deviation

$$
S_{r}=\sqrt{\frac{1}{N-1} \sum_{k=1}^{N}\left(r_{k}-\bar{r}\right)^{2}}
$$

The standard deviation of the mean of the results, $\bar{r}$ is given as 


$$
S_{\bar{r}}=\frac{S_{r}}{\sqrt{N}}
$$

The random uncertainty calculated directly from the result inherently includes the effects of all correlated random error sources.

The total uncertainty of the result, $U_{r}$ with $95 \%$ confidence is then the root-sum-square of the systematic and random error contributions such that

$$
U_{r}=\sqrt{B_{r}^{2}+\left(2 S_{r}\right)^{2}}
$$

or of the mean of the results, $U_{\bar{r}}$

$$
U_{\bar{r}}=\sqrt{B_{r}^{2}+\left(2 S_{\bar{r}}\right)^{2}}
$$

Notice $U_{\bar{r}}$ quickly approaches the systematic error as $N$ approaches infinity. Coleman and Steele suggest a minimum of 10 samples is necessary to estimate a $95 \%$ confidence limit with $2 S_{r}$.

\section{General Error Sources and Paths}

Static and forced oscillation wind tunnel tests were conducted at the 14- by 22-Foot Subsonic Tunnel at NASA Langley Research Center. Two aircraft configurations were tested (Fig. 1) including a cranked-arrow wing fighter aircraft configuration and a Generic Civil Transport (GCT) configuration (Fig. 2 and Fig. 3 respectively). Data were obtained over a broad range of $\alpha$ 's, motion amplitudes, and frequencies of oscillations. Data were sampled at 200 $\mathrm{Hz}$ for various durations of time with $100 \mathrm{~Hz}$ anti-aliasing filters. Post-test digital filtering was also used for analysis of the data and will be discussed where applicable.

Wind tunnel test procedures and instrumentation vary with facility, test engineer, and certainly with the test objectives. However data reduction procedures used to estimate aerodynamic coefficients for static and forced oscillation are conceptually similar. Balance and attitude/orientation measurements are recorded first with the wind off. These measurements are collectively called tares. They are used to subtract weights and inertial effects from the forces and moments recorded with the wind on. Data are acquired from the data acquisition system as voltages. The data acquisition system contributes its own uncertainties to the voltages output from the balance and attitude/orientation instruments. The acquired voltages are then sent to a computer for conversion to engineering units, corrections, interpolations, and data reduction. Each one of those steps contributes error as well as round-off error from operating in the digital environment. Round-off error will be considered negligible relative to other error sources and will not be quantified; however it is important to note all computations are in a finite digital domain.

Errors from data acquisition in the tare and wind-on runs combine with errors from the balance sensitivities, interaction coefficients and model attitude/orientation sensitivities. Tares are typically recorded at attitudes/orientations with a slightly larger range but fewer set points than wind-on runs for static tests and thus tare values will be interpolated for data reduction. Forced oscillation tests are similar in that the tare must be interpolated, however this is due to sampling and will be discussed in detail later. Interpolations are treated as any other data reduction function. As such the uncertainties from tare forces and moments (ordinate) and tare attitudes/orientations (abscissa) combine with uncertainties from the wind-on run attitudes/orientations to give a final uncertainty in the interpolated forces and moments. The uncertainty from the data acquisition system also combines with errors in the pressure transducer sensitivity and dynamic pressure correction uncertainties to yield a final uncertainty on dynamic pressure.

Figure 4 illustrates how error sources from tares and wind on runs combine with sensitivities of instruments (calibration regressions), interpolations and finally into the data reduction equation to produce uncertainty on the body axis aerodynamic coefficients. Blue and green are used to represent error paths of tare measurements and wind on run measurements respectively. Error sources are listed in rounded rectangles. Red is used to illustrate combined error from both tare measurements and wind on run measurements. 


\section{Static Test Results}

Consider the uncertainty propagation through the body axis coefficient equations. Equation (10) is a generic body axis coefficient equation where $X$ represents the aerodynamic force or moment of interest and $K$ is the corresponding nondimensionalizing multiplier (S, $S b$, or $S \bar{c}$ where appropriate). Assuming $K$ has negligible uncertainty, the uncertainty of the coefficient can be found from Eq (11). Note the dynamic pressure, $q$ in the denominator of both terms. One could infer testing at a higher dynamic pressure would be one way to reduce uncertainty. Unfortunately adverse model dynamics due to sting vibrations and lower resolution balances often accompany larger dynamic pressures, which in turn increases the uncertainty of the aerodynamic force or moment, $U_{X}$.

$$
\begin{gathered}
C_{X}=\frac{X}{q K} \\
U_{C_{X}}^{2}=\left(\frac{\partial C_{X}}{\partial X}\right)^{2} U_{X}^{2}+\left(\frac{\partial C_{X}}{\partial q}\right)^{2} U_{q}^{2} \\
=\left(\frac{1}{q K}\right)^{2} U_{X}^{2}+\left(\frac{C_{X}}{q}\right)^{2} U_{q}^{2}
\end{gathered}
$$

The uncertainty of all balance loads for both models varies with the angle-of-attack. As one would expect, the variance of the forces and moments increase with $\alpha$ and peak near stall as the flow transitions from fully attached to fully separated. The flow in this transition region often induces adverse dynamics in the support structure, balance and the model. Figure 5 and Fig. 6 are the results of the uncertainty propagation for the body axis aerodynamic coefficients of normal force and pitching moment respectively of the fighter model. The range between the upper and lower $95 \%$ confidence limits (green error bars) near stall are nearly $25 \%$ of the overall range of the normal force coefficient (Fig. 5). The majority of uncertainty in the normal force coefficient after filtering (red error bars) at $1 \mathrm{~Hz}$ is due to the systematic error or bias as shown by the blue error bars. The majority of the bias in normal force coefficient arises from the dynamic pressure calibration of the wind tunnel test section as the biases from the balance in the run and tare are assumed perfectly correlated and cancel out. The same trend can be seen in all other coefficients but it is not so dramatic as with normal force because the other coefficients are much smaller than unity. Thus the unsteady contribution from the balance signals dominates the uncertainty of all other coefficients as seen with the pitching moment (Fig. 6) where the spread between the blue error bars is a much smaller fraction of the span of the red error bars.

\section{Dynamic Test Results}

Forced oscillation tests use the same internal strain-gauge balance as used in static testing to measure the forces and moments of the model. The major difference between static testing and forced oscillation for determining aerodynamic loads is the addition of inertial loads created by the oscillations, which need to be removed in addition to gravity tares. Models oscillate and vibrate during static tests, however models are routinely forced to sinusoidally oscillate at amplitudes up to $30^{\circ}$ at frequencies greater than $1 \mathrm{~Hz}$ in dynamic tests. Traditionally data is sampled at $200 \mathrm{~Hz}$ for 40 sinusoidal periods or 40 cycles. The data is then digitally low-pass filtered at $4 \mathrm{~Hz}$. A few runs for this test were sampled for over 120 cycles to observe how the mean cycle and standard deviation change with additional cycles. Variable frequencies and amplitudes with a fixed sample rate give rise to variable position samples, meaning samples are not recorded at the same cycle position for each cycle. For this reason $n$ points from 0 to $2 \pi$ around each cycle are interpolated and then averaged down to one mean cycle.

As with the static test, low $\alpha$ 's typically have low uncertainties. The unsteady aerodynamics usually increase to a maximum around stall and then decrease post-stall. Figures 7 and 8, yaw oscillations of the fighter model at $\alpha=10^{\circ}$ and $50^{\circ}$ respectively, illustrate the change in variation from cycle to cycle with angle-of-attack. Coefficient data for all 120 interpolated continuous cycles, the mean cycle, and the bias are shown. Note difference between the read and blue error bars shows that the largest contribution to the total uncertainty in both cases is due to the inherent unsteady aerodynamics and structural vibrations that were not filtered out. Figure 8 shows large variations from cycle to cycle. At $50^{\circ}$ angle-of-attack the wing is stalled and the vertical tall is completely blanketed. The resulting 
mean does not represent the high degree of unsteadiness at this condition. Roll oscillations at $\alpha=4^{\circ}$ and $50^{\circ}$ are shown in Fig. 9 and Fig. 10 respectively for the GCT model. The higher amplitude of this oscillation, $20^{\circ}$, results in larger variations of states across the cycle, which contributes to data showing cleaner trends than seen with the small, $5^{\circ}$, amplitude oscillation of the fighter at $50^{\circ}$.

The uncertainties indicate the statistical confidence in the data and not really the "validity" of the data. The data validity has to do with models and variables inherent in the data that might not be under control or observation, and experience has shown that the means of the data tend to repeat fairly well from test to test, even though the cycles show large variation between each other. This is a method for putting an estimate of certainty on the data, cycle to cycle (not total mean), based on the data and measurements taken, and therefore can be used for developing design margins, etc.

\section{Sample Duration Requirements}

Collecting forced oscillation data tends to be very time intensive, and therefore costly, so a method to determine the amount of data needed to arrive at a "good" value without continuing to take more data than needed is a valuable improvement. The mean and the standard deviation of a sample from a Gaussian distribution will approach the true mean and true standard deviation as the number of readings in that sample approaches infinity. These mean and standard deviation histories, which are continuously recalculated as new readings are recorded, will be defined as the "rolling mean" and "rolling standard deviation". The differences between successive rolling means and rolling standard deviations or their gradients will generally decay as the number of readings approaches infinity. The gradients may oscillate in sign, as the random error is equally likely to be positive or negative, but the overall decay of the absolute value of the gradients will be similar to an asymptotic decay.

Consider the gradients of the rolling standard deviation for all 100 points around the cycle in Fig. 10. Every point will have a different gradient from cycle to cycle. The angular position, $\theta$ on the cycle at which the maximum gradient occurs at cycle 1 may be different at cycle 2 and so on. That maximum gradient can be used to identify how much the uncertainty of the entire cycle is changing from cycle to cycle. Now consider a 10-cycle moving average of the maximum gradients, $g_{m}$ (Fig. 11). The gradient of the mean is also shown for reference but is not used as a stopping criterion. A moving average was necessary to smooth the maximum gradients. Ten cycles were chosen for the moving average so the precision limit could be estimated with $95 \%$ confidence by $2 S_{r}{ }^{2}$ Notice, at the scale presented, the gradients level off around 60 cycles. A threshold could be set such that if a gradient crosses a prescribed tolerance there is no need to continue collecting data at that condition. The bias of the balance, for example would be a good tolerance limit. However there is no guarantee a threshold will be met especially when a new model is tested. Some flexibility needs to be built into the stopping criteria.

The objective is to stop when the mean and uncertainty (or standard deviation since the bias is fixed) stops changing significantly. It is proposed a threshold be set that is $10 \%$ of the average rate of change of the uncertainty after 11 cycles. The 11-cycle wait is needed because the standard deviation of the first cycle is zero. This would provide a metric, which is case specific, provides an upper bound on the uncertainty around the cycle, and is sensitive to variation of the mean. If the variation in the cycles is small, such as in the linear region of the lift curve slope, the $10 \%$ threshold will be very low. To prevent from sampling forever to reduce the already low values, another threshold is needed, and use of the known balance accuracies is proposed. The balance accuracy as a function of load was not available. However, experience has shown that balance measurements can be made to at least half of the full-scale accuracy reported by the calibration laboratory. Thus half the listed balance accuracy will be used as a fixed threshold. The final data acquisition stopping criteria is achieved when $g_{m}$ is $10 \%$ of the gm after the initial 11 cycles or less then half of the balance accuracy. Data acquisition continues until these criteria are satisfied for all balance components. This process is outlined in Fig. 12.

The stopping criteria presented applies to all corrected balance loads, i.e. after interactions are removed. The stopping criteria are not met at the same time for all balance loads. Thus the last load to meet the terminating conditions will terminate sampling and then the model can move to the next test point. The yaw oscillations for the fighter model could have ended after 11 cycles for the tare and $11,38,37,48$ and 40 cycles at $\alpha=10^{\circ}, 38^{\circ}, 40^{\circ}, 50^{\circ}$ and $60^{\circ}$ respectively (Fig. 13). Figure 14 is an example of how this technique can be used for post-analysis of standard 40-cycle data to see where more cycles were needed and where more cycles were recorded than necessary. Green is used to indicate when the stopping criteria was meet before the cycle limits, 40-cycles, where reached. Red indicates when the stopping criteria was not reached before reaching the end of the recorded data cycles. The first and last 450 points in the 40 -cycle time-histories were removed to remove any transients from filtering. This is why the cycle limits do not reach exactly 40 in some cases, but do reach the total number of complete cycles. As one would expect more data is acquired than needed in the linear region and not enough near and post stall.

6

American Institute of Aeronautics and Astronautics 


\section{Conclusions}

A procedure has been developed and tested to estimate the uncertainty associated with dynamic aerodynamic data obtained from forced oscillation tests in a wind tunnel. This technique can be used to assess the confidence in the measured data, and to guide data acquisition to ensure enough data samples are taken to arrive at meaningful conclusions. This process can be applied to oscillatory dynamic data as well as to static data where unsteady effects or other processes cause variations in balance measurements at static conditions. The procedures allow for a basis with which to design flight controls in the presence of uncertainties and give the user unique insight to the data, which is otherwise not available.

\section{References}

${ }^{1}$ Slotine, J. E., and Li, W., Applied Nonlinear Control, Prentice Hall, Englewood Cliffs, 1991.

${ }^{2}$ Coleman, W. H., and Steele, W. G., Experimentation and Uncertainty Analysis for Engineers, ${ }^{\text {nd }}$ ed., John Wiley and Sons, New York, 1999.

${ }^{3}$ Heim, E. H. D., "Development of Methods for Improved Data Integrity and Efficient Testing of Wind Tunnel Models for Dynamic Test Conditions in Unsteady and Nonlinear Flight Regimes," M.S. Thesis, Aerospace and Ocean Engineering Dept., Virginia Polytechnic Institute and State University, Blacksburg, VA, 2003.

${ }^{4}$ Murphy, P. C., and Klein, V., Estimation of Aircraft Unsteady Aerodynamic Parameters from Dynamic Wind Tunnel Testing, AIAA-2001-4016.

${ }^{5}$ Shah, G. H.; Cunningham, K.; Foster, J.V.; Fremaux, C. M.; Stewart, E. C.; Wilborn, J. E.; Gato, W.; and Pratt, D. W., Wind-Tunnel Investigation of Commercial Transport Aircraft Aerodynamics at Extreme Flight Conditions, SAE-2002-01-2912.
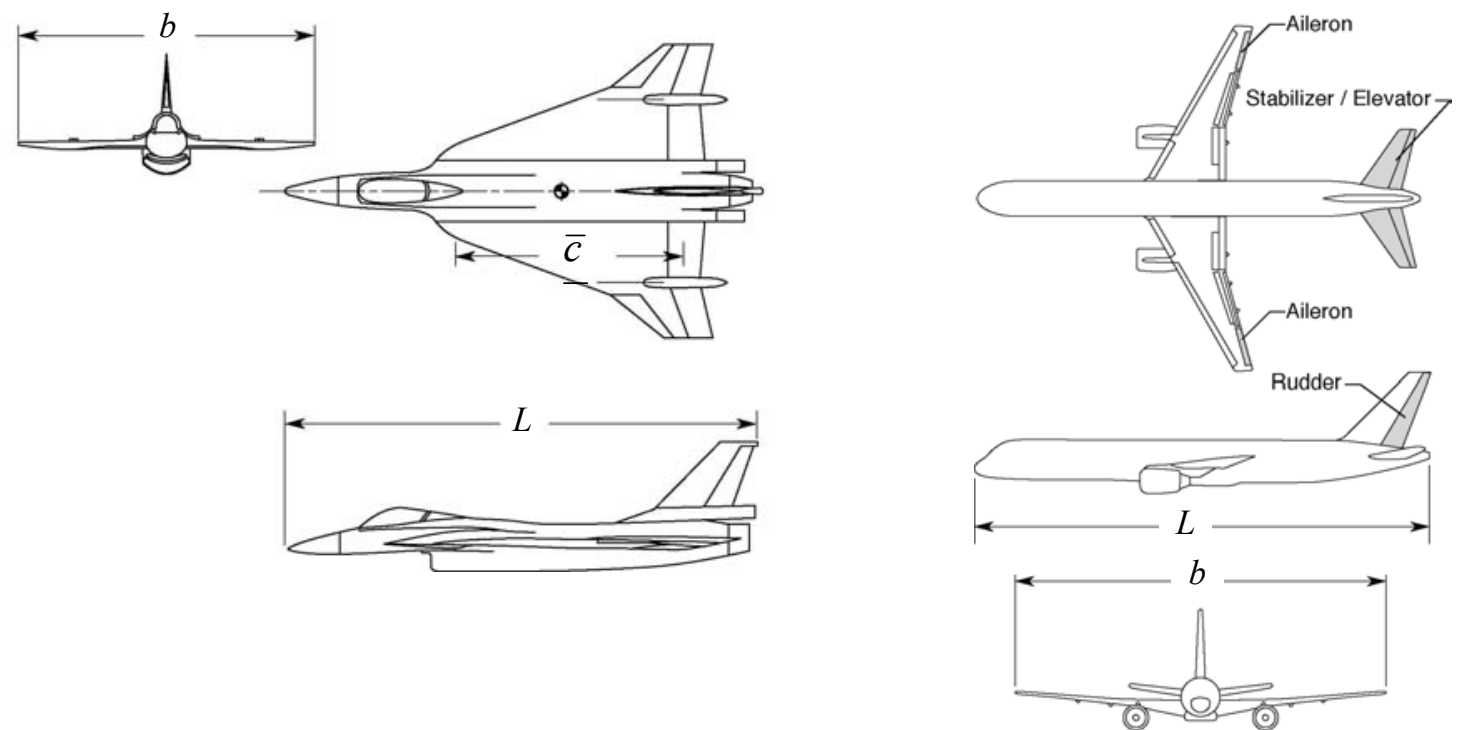

$\begin{aligned} S & =19.44 \mathrm{ft}^{2} \\ b & =5.83 \mathrm{ft} \\ \bar{c} & =4.45 \mathrm{ft} \\ L & =9.76 \mathrm{ft}\end{aligned}$

(a)

$\begin{aligned} S & =5.90 \mathrm{ft}^{2} \\ b & =6.85 \mathrm{ft} \\ \bar{c} & =0.92 \mathrm{ft} \\ L & =8.54 \mathrm{ft}\end{aligned}$

(b)

Figure 1. Schematics of (a) fighter model and (b) generic civil transport model. ${ }^{3,4}$ Note drawings are to scale with respect to one another. 


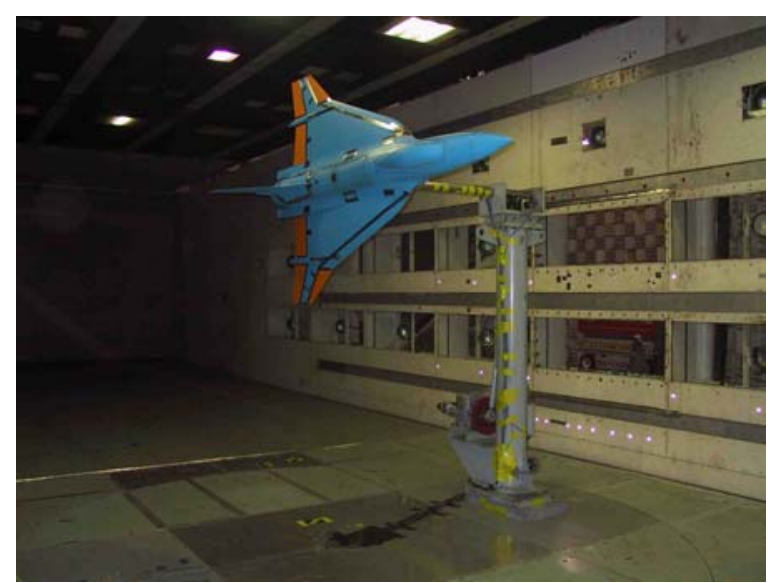

Figure 2. Fighter model mounted in wind tunnel for yaw oscillation tests.

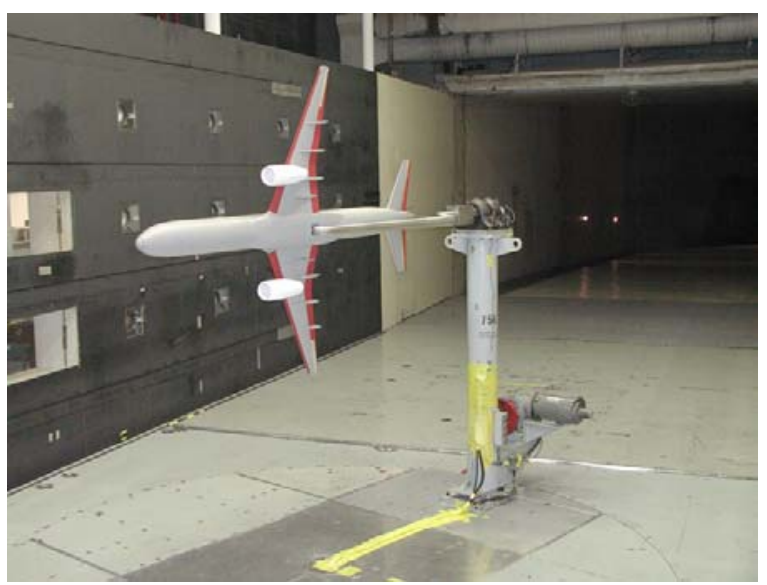

Figure 3. GCT model mounted in wind tunnel for roll oscillation tests.

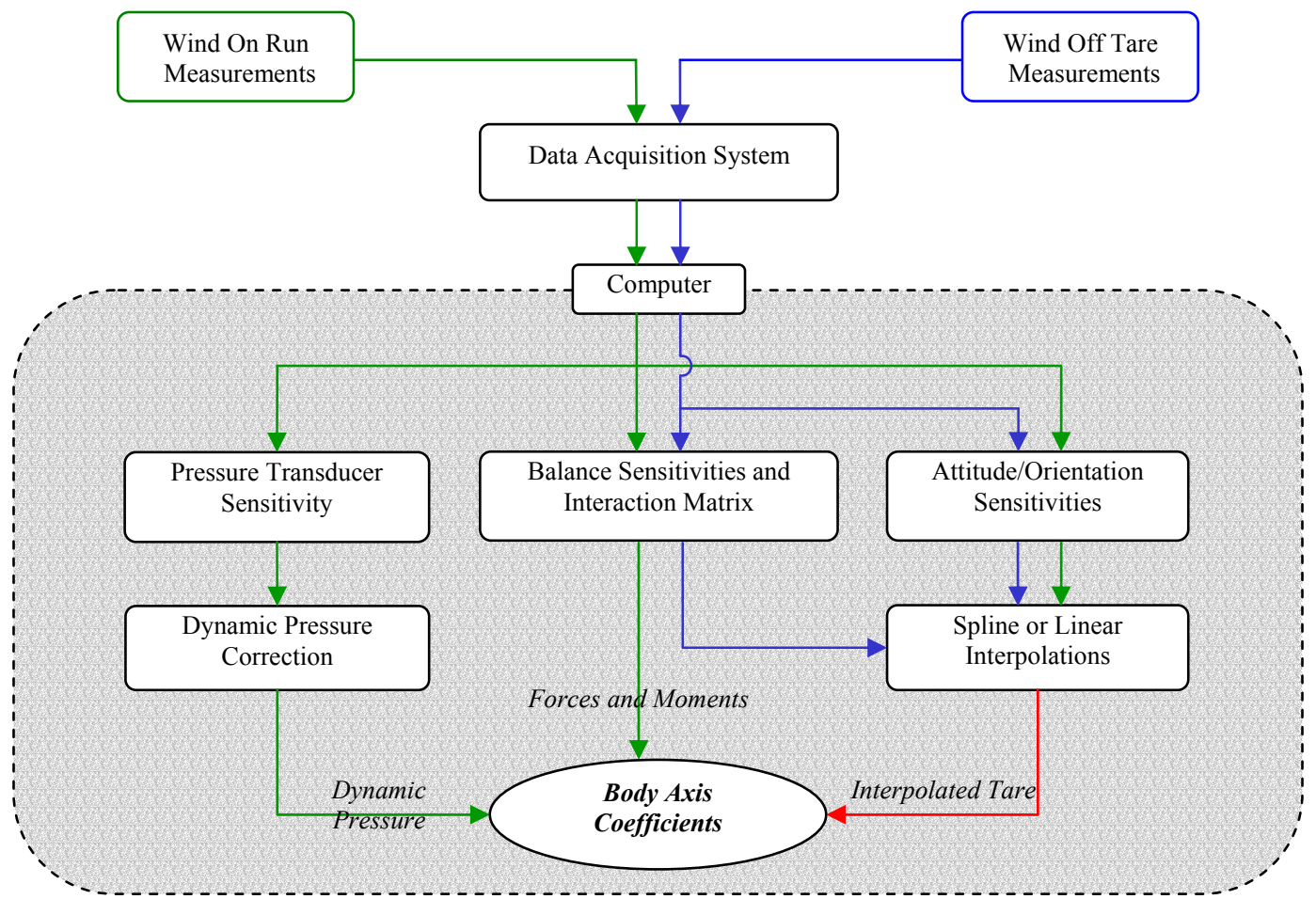

Figure 4. Error sources and paths from wind on run measurements and wind off tare measurements that contribute to aerodynamic coefficient uncertainties. 


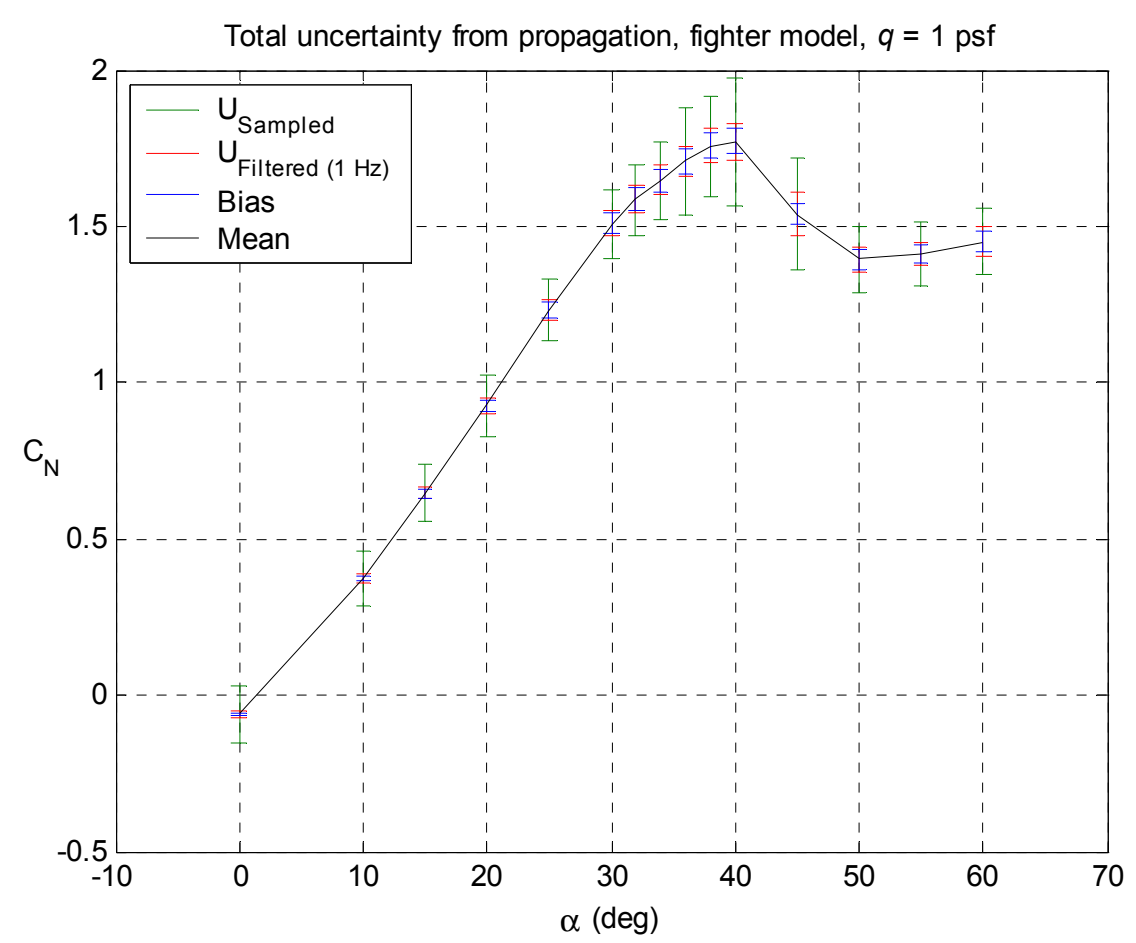

Figure 5. Total uncertainty of normal force coefficient from propagation method for sampled and postfiltered data verses the bias contribution.

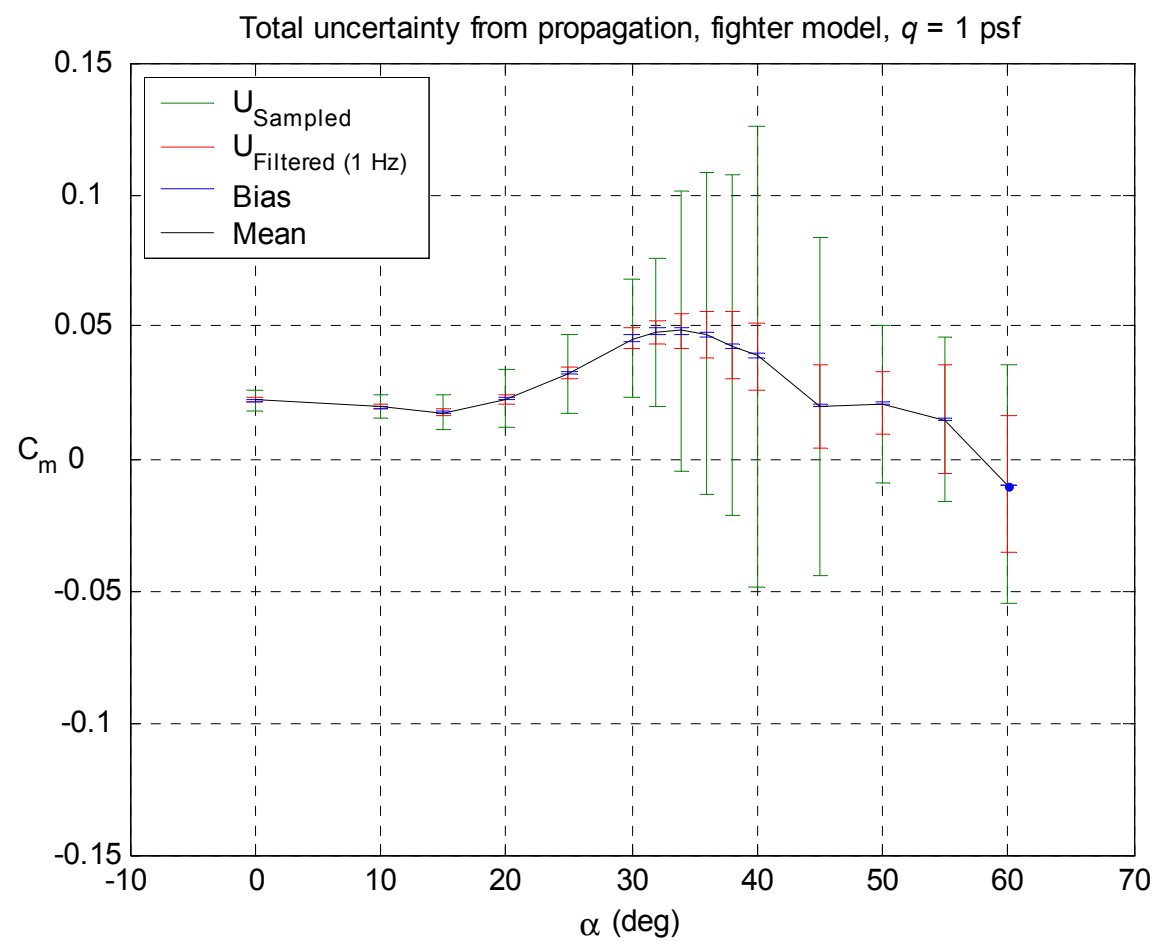

Figure 6. Total uncertainty of pitching moment coefficient from propagation method for sampled and postfiltered data verses the bias contribution. 


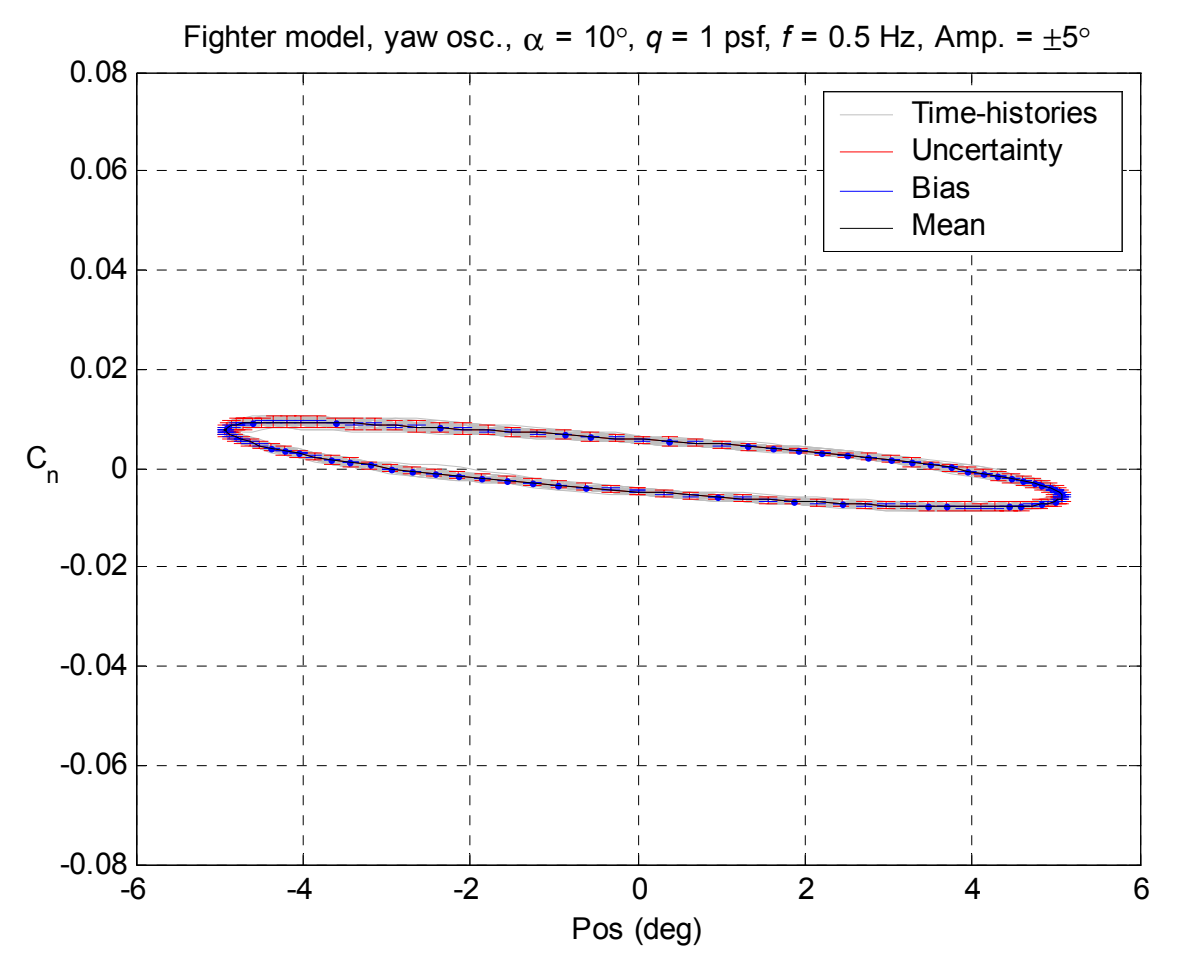

Figure 7. Time-history of 120 cycles oscillated at $0.5 \mathrm{~Hz}$ for yawing moment coefficient.

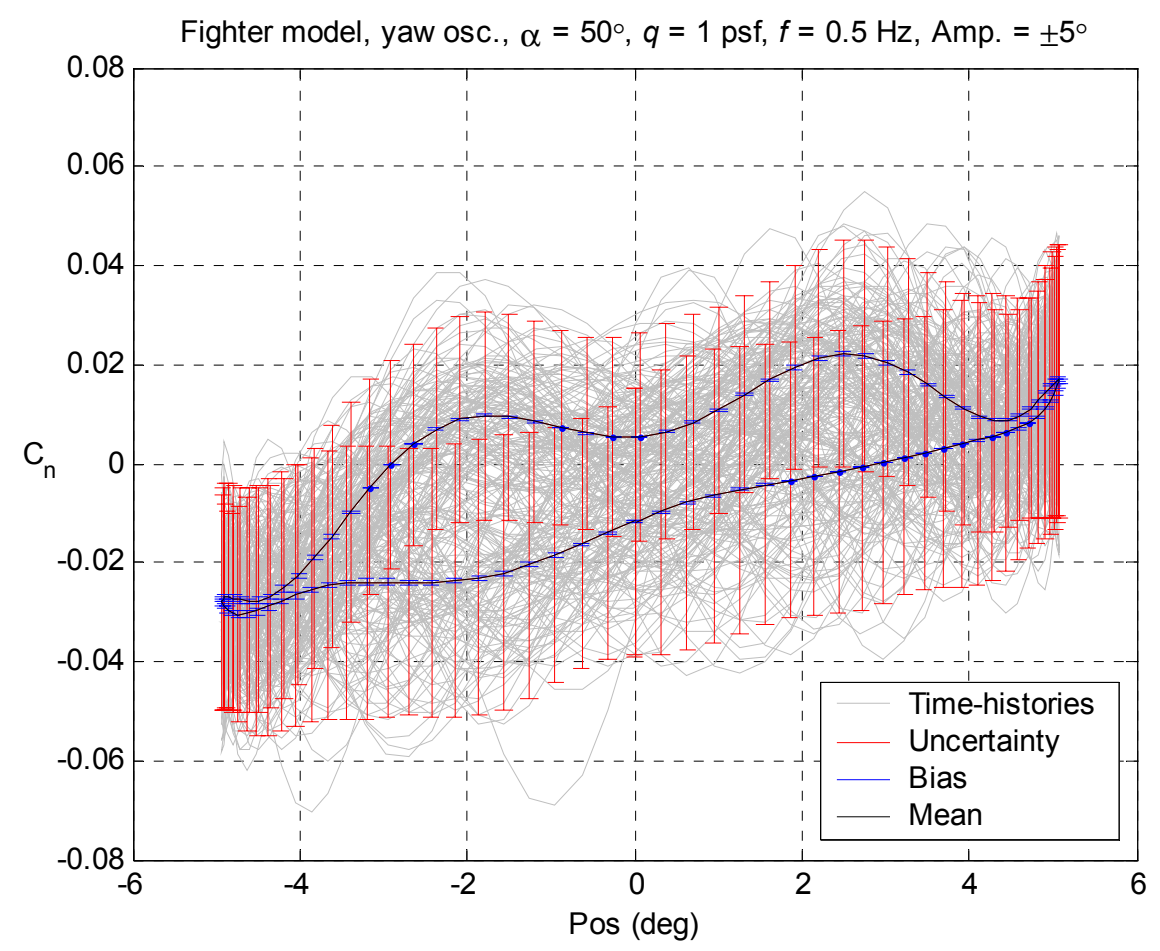

Figure 8. Time-history of 120 cycles oscillated at $0.5 \mathrm{~Hz}$ for yawing moment coefficient. 


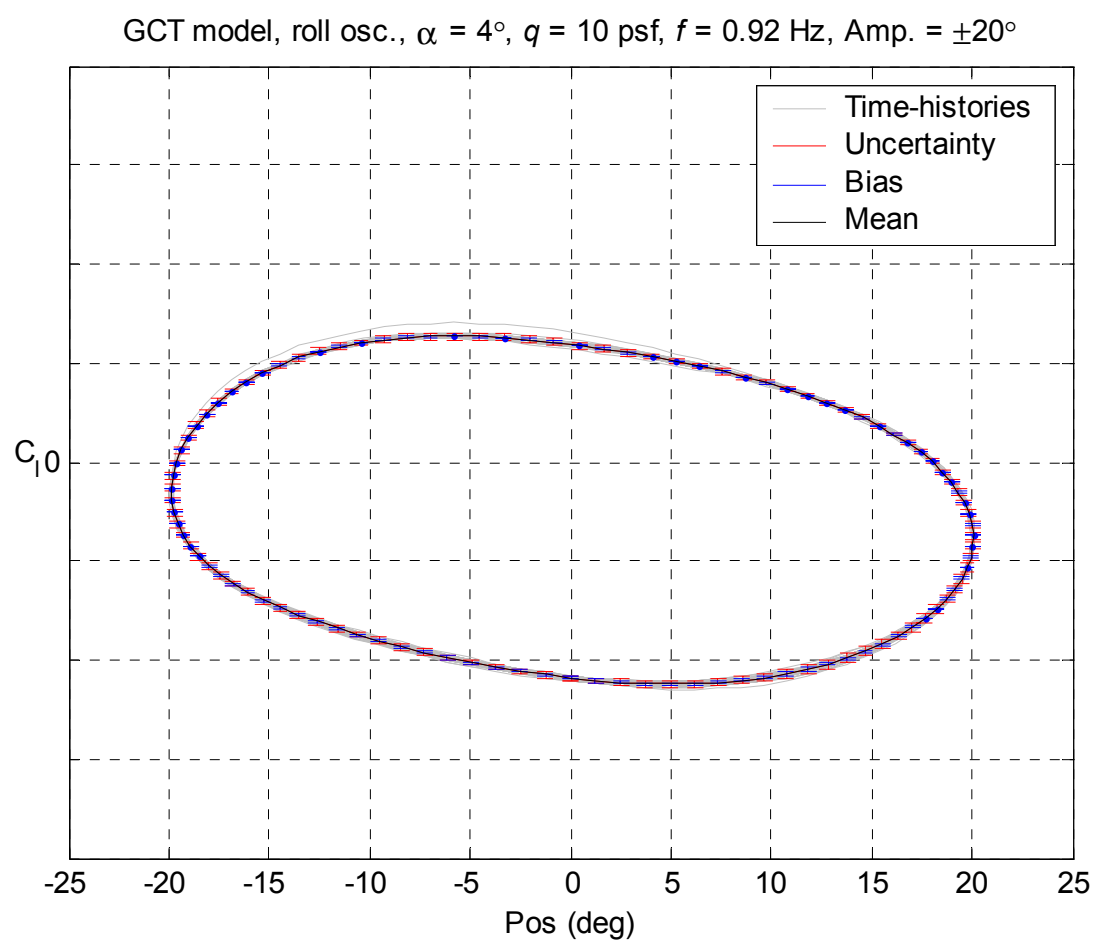

Figure 9. Time-history of 120 cycles for GCT oscillated at $0.92 \mathrm{~Hz}$ for rolling moment coefficient at $\alpha=4^{\circ}$.

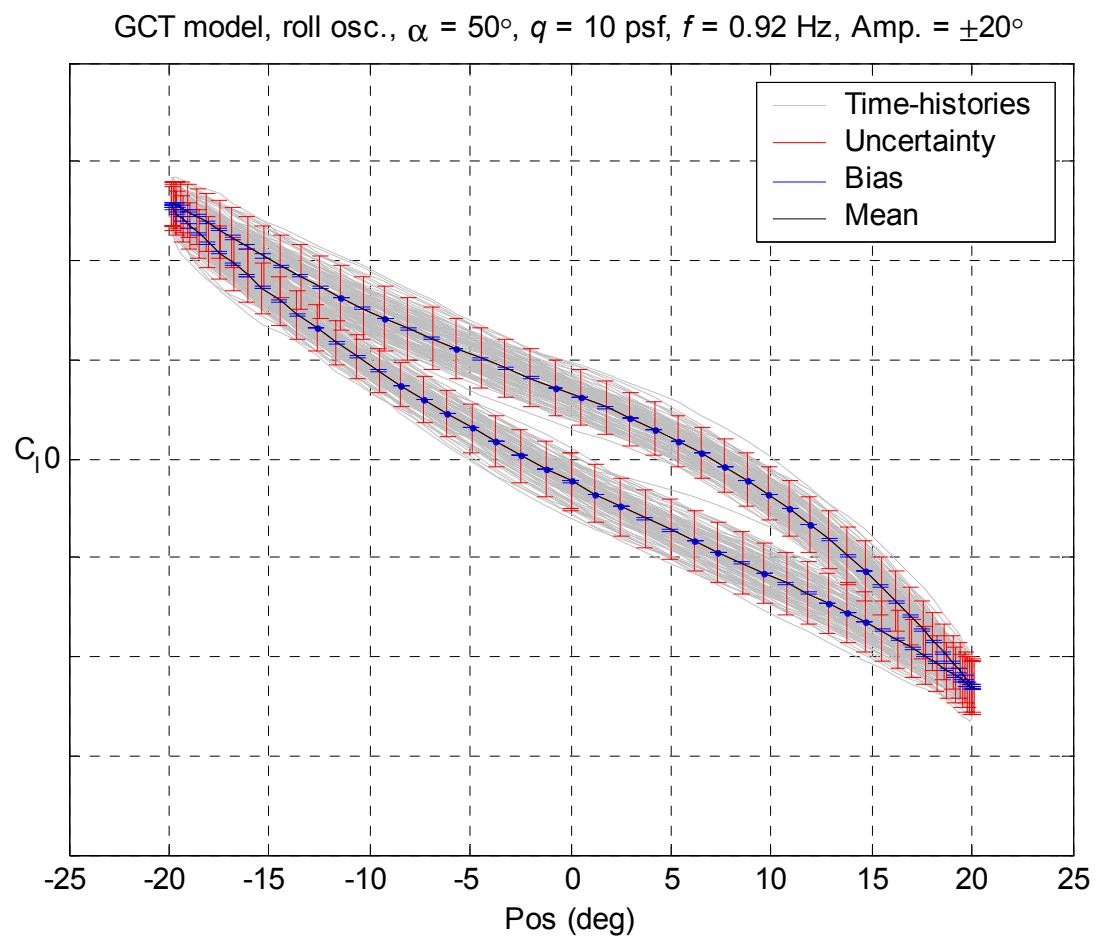

Figure 10. Time-history of 120 cycles for GCT oscillated at $0.92 \mathrm{~Hz}$ for rolling moment coefficient at $\alpha=$ $\mathbf{5 0}^{\mathbf{6}}$. 


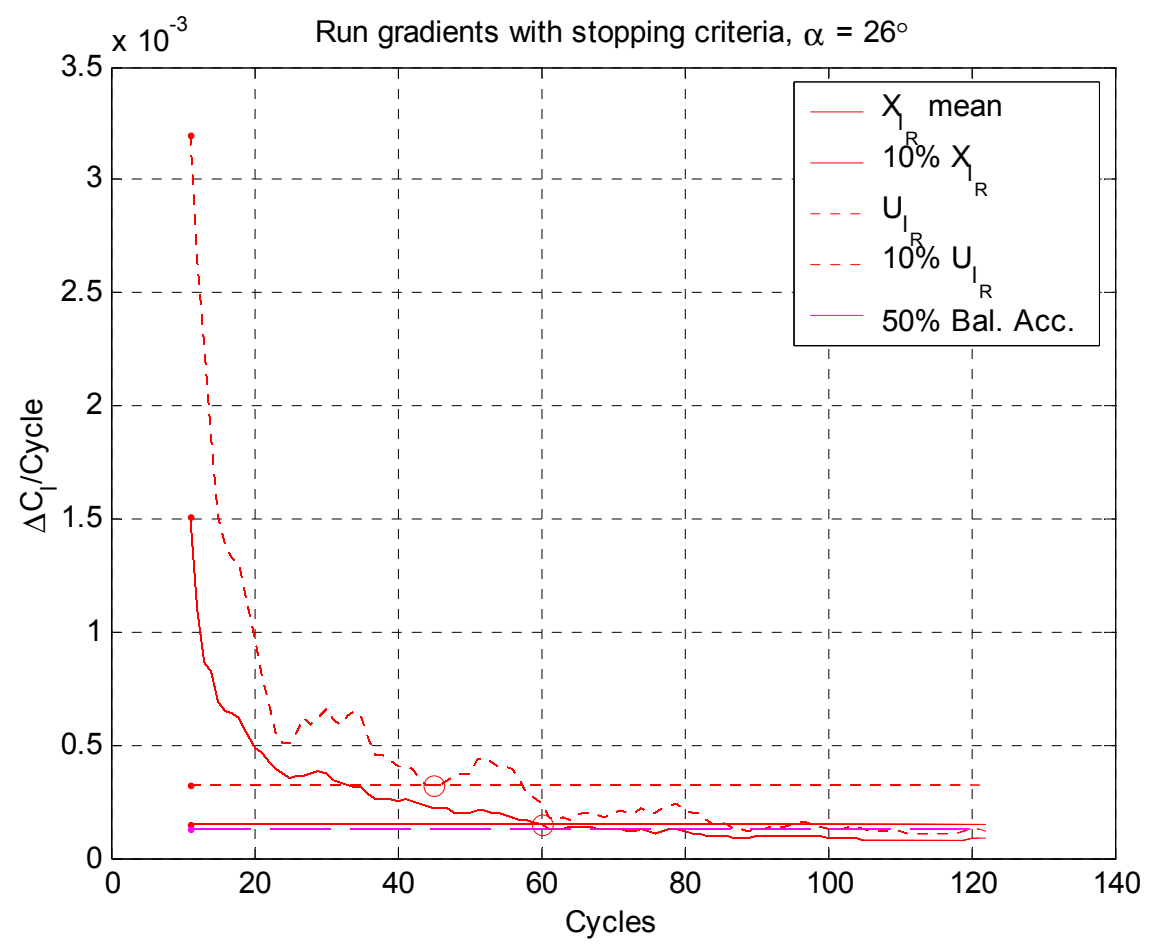

Figure 11. Time history of stopping criteria for rolling moment at 26 degrees for 121 cycles. Circles indicate when cycle stopping criteria is met.

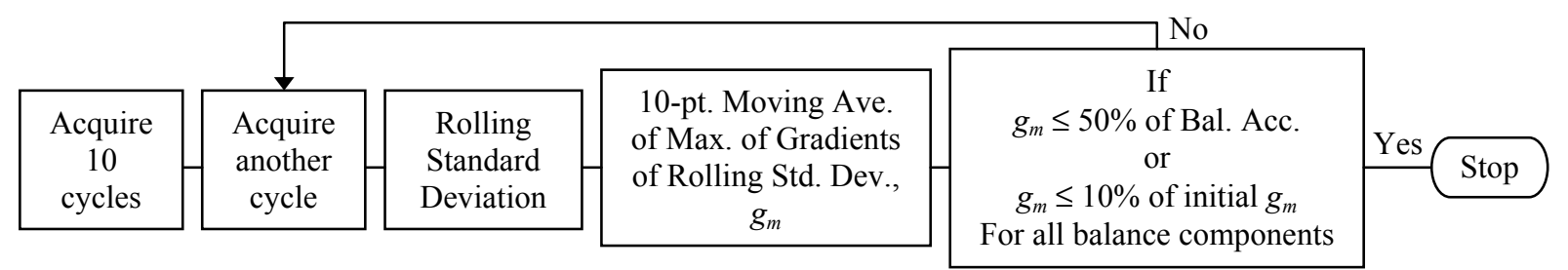

Figure 12. Flowchart of forced oscillation data acquisition termination process.

12

American Institute of Aeronautics and Astronautics 


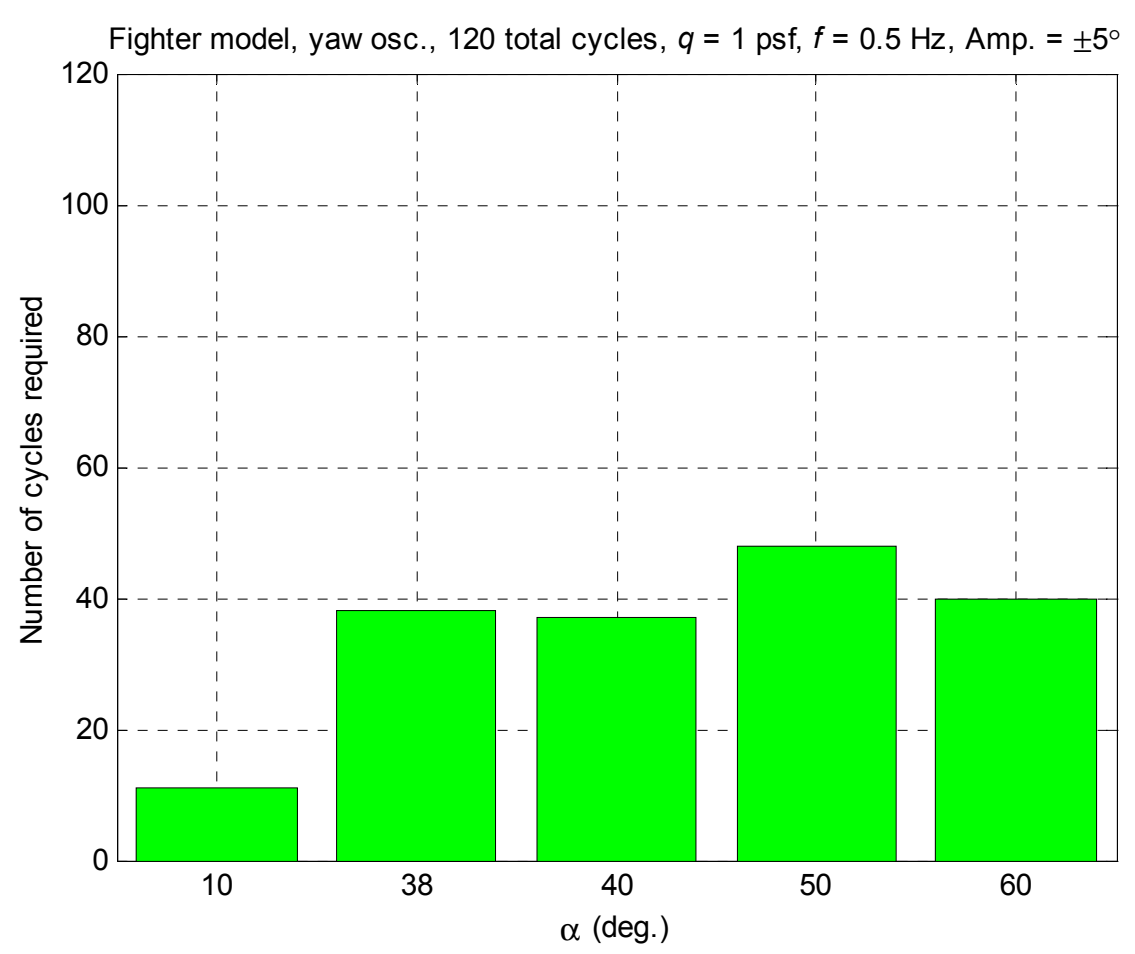

Figure 13. Cycles required for fighter model using stopping criteria.

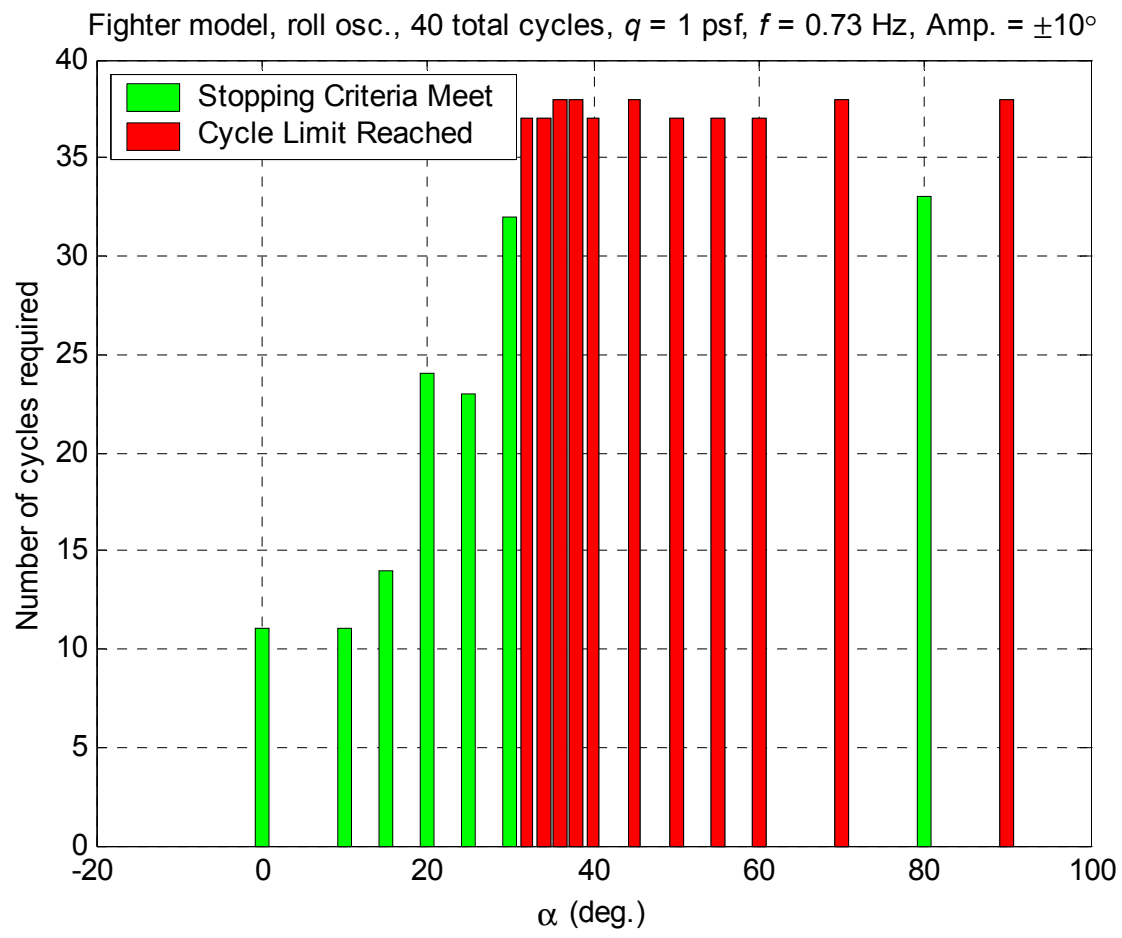

Figure 14. Example of post-analysis for fighter model. Note cycle limits are less than 40 and vary due to filter transience. 